\title{
Tracking and Analysis of Cine-Delayed Enhancement MR
}

\author{
Thomas O’Donnell ${ }^{1}$, Engin Dikici ${ }^{1}$, Randolph Setser ${ }^{2}$, and Richard D. White ${ }^{2}$ \\ ${ }^{1}$ Siemens Corporate Research, 755 College Road East, Princeton, NJ 08540 \\ ${ }^{2}$ Cleveland Clinic Foundation, Division of Radiology, 9500 Euclid Ave., \\ Cleveland, $\mathrm{OH}, 44195$ \\ tom. odonnell@siemens.com
}

\begin{abstract}
Cine-DEMR is a new cardiac imaging technique which combines aspects of Cine and Delayed Enhancement MR. Like Cine, it displays the heart beating over time allowing for the detection of motion abnormalities. Like DEMR, non-viable (dead) tissues appear with increased signal intensity (it has been shown that the extent of non-viable tissue in the left ventricle (LV) of the heart is a direct indicator of patient survival rate). We present a technique for tracking the myocardial borders in this modality and classifying myocardial pixels as viable or non-viable. Tracking is performed using an affine deformed template of borders manually drawn on the first phase of the series and refined using an ASM-like approach. Classification employs a Support Vector Machine trained on DEMR data. We applied our technique on 75 images culled from 5 patient data sets.
\end{abstract}

\section{Introduction}

Cine-Delayed Enhancement Magnetic Resonance imaging (Cine-DEMR) is a novel imaging technique targeted to the left ventricle of the heart which combines the advantages of both Cine MR and Delayed Enhancement MR (DEMR). Like Cine imaging, Cine-DEMR recovers the motion of the heart over the cardiac cycle - the detection of motion abnormalities such as hypokinesis in Cine is a well established indicator of cardiac health [Schwartzman]. Like DEMR images, non-viable (dead) myocardial tissue appears bright in Cine-DEMR images, allowing the amount of nonviable myocardium to be quantified - the extent of non-viable tissue in DEMR is directly correlated with improved cardiac function after revascularization therapy (e.g. coronary bypass surgery) [Kim NEJM]. Thus, Cine-DEMR unites morphology (Cine) with function (DEMR). Figure 1 shows sample images from a Cine-DEMR study and compares them to Cine and DEMR images.

Cine-DEMR has advantages over the combination of separate Cine and DEMR acquisitions. First, it decreases scanning time by replacing two acquisitions with one no small benefit given the costs of imaging. Second, it supplants the mental integration of the two sequences with a perfectly fused simultaneous visualization. Third, it avoids potential mis-registration of the two separate sequences. Cine and DEMR are typically acquired minutes apart. During that time the patient may have moved, or chest positions may be at variance due to differing breath intakes. The 
resulting images may describe regions millimeters apart. Fourth, the optimal phase for evaluating scar (non-viable) tissue may not be end-diastole (the phase in which DEMR is typically acquired). Fifth, due to motion of the myocardium normal to the imaging plane as the heart contracts, the scar may appear and disappear. With CineDEMR, the phase which best describes the scar is much more likely to be imaged simply because more than one phase is acquired. Finally, in cases where the scar does not completely cover the wall (is not transmural), it is easier to see how the healthy portion moves as compared to Cine because the two regions can be distinguished.

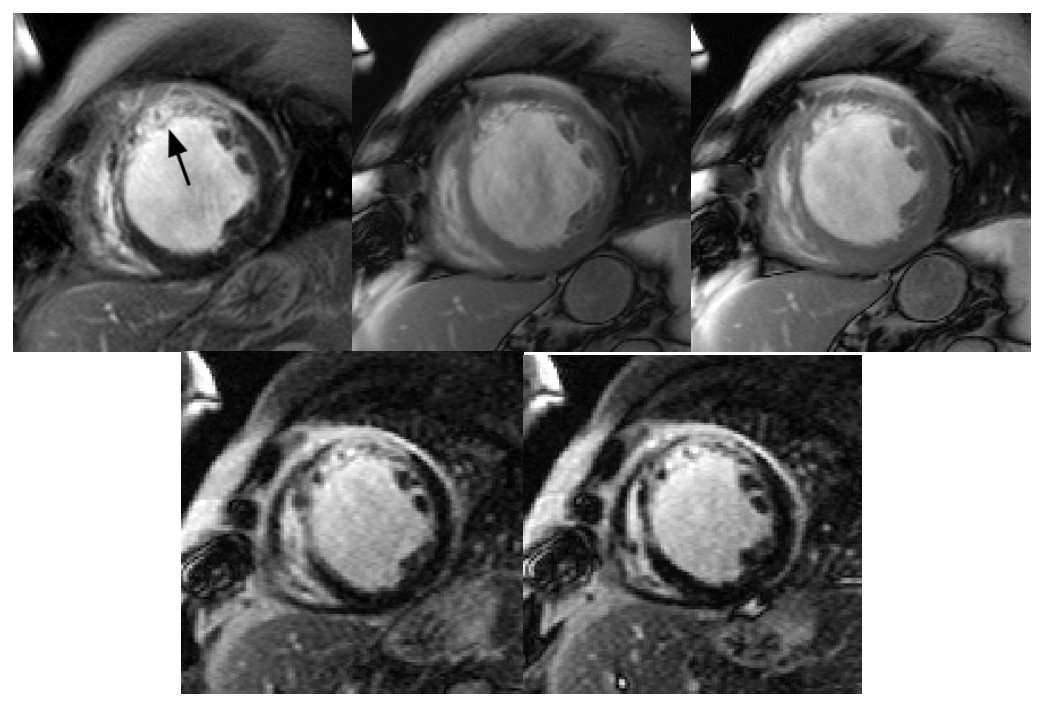

Fig. 1. Top Left: A DEMR image showing non-viable regions with increased signal intensity (denoted by arrow) (Note: DEMR images are single phase). Top Middle and Right: Enddiastolic and end-systolic images from a mid-ventricular short-axis Cine acquisition. Bottom: Images from a 15 phase Cine-DEMR study. End-diastole is on the left, end-systole on the right.

The above benefits, however, come at a cost. Cine-DEMR has both decreased spatial and temporal resolution compared to the individual sequences. Nevertheless, preliminary studies in which clinicians analyzed Cine-DEMR, Cine, and DEMR images of the same individual have shown showed a strong correlation in the categorization of tissues [Setser]. And, work is currently being done to improve the temporal resolution via parallel imaging and/or segmented K-space schemes [Setser].

The ultimate goal of our work is the quantification of myocardial scar in CineDEMR images. By quantification we mean the identification and measurement of dead myocardial tissue. This implies a two part analysis scheme. First, the myocardium must be defined via the delineation of its borders. Then, those myocardial tissues must be classified as viable or non-viable.

The first part of the scheme, the delineation of the myocardium, is quite challenging. Compared to the same task in Cine MR, it is harder since the myocardial borders may be completely missing. That is, the signal of the myocardium may be the 
same as that of the bloodpool in non-viable regions. And, since the scar may appear and disappear due to thru plane motion we cannot guarantee that the same intensity pattern will be present over the cycle. Compared to the task in DEMR, it is also difficult. Previous quantification schemes for DEMR [Dikici] assumed the existence of a Cine study so that the true morphology could be referenced. With the advent of Cine-DEMR there may not be a separate Cine study.

Thus, to simplify the delineation task, we assume the existence of a manually drawn contour on the first frame and track the borders over the cycle using this starting point. Briefly, we register this first frame to each of the subsequent (target) frames, copy the endo and epi contours over and fit them to the edges in the target frame using affine transforms. To refine the fit, we take an active shape model approach by attempting to match the radial intensity profiles in the target image to those in the first frame. A spline is then fit to the individual contour points to smooth them. For the second part of the scheme, the classification of the tissues, we rely on a Support Vector Machine (SVM) [Cristianini] which has been trained to recognize non-viable tissue in DEMR images.

The remainder of this paper is as follows. In Section 2 we describe related work. In Section 3 we provide a more detailed description of our algorithms. In Section 4 we show results on the tracking and analysis of Cine-DEMR studies from 5 patients with 15 frames each for a total of 75 images. Discussion of these results are presented in Section 5 and finally, in Section 6 we submit our conclusions.

\section{Related Work}

While both Cine and Delayed Enhancement MR have become standard protocol for assessment of cardiac viability when using MR [Schwartzman], Cine-DEMR is new enough that, to our knowledge, no work has been published on its automatic or semiautomatic analysis.

Dikici et. al. [Dikici], segmented static DEMR on a slice by slice basis using a standard Cine image set as a morphology reference. The employment of an affine deformed template for the tracking of the myocardium was done by [Sun] which bares resemblance to our initial tracking scheme. Our refinement of the tracking uses an active shape model [Cootes] approach similar to that of [Mitchell], however, it is not "trained" on a set of images. Rather, we simply use the first frame of the series as a comparison.

Regarding the classification of myocardial tissues using DEMR, Noble et. al., registered Cine and DEMR image sets in $3 \mathrm{D}$ in order to detect hibernating (dormant but not dead) myocardium [Noble]. As well, there are several semi-automatic segmentation techniques for classifying DEMR published in the literature [Kim]. Some of these studies have defined a threshold for non-viable pixels as $>2 *$ std dev of the signal intensity of remote, viable myocardium (which was defined manually) [Kim]; other studies have used $3 *$ std $\operatorname{dev}$ as the threshold [Fieno]. We employ a Support Vector Machine to classify tissues and in this regard are similar to El-Naqa et. al., in their work on mammogram analysis [El-Naqa] and Dikici et. al.,[Dikici] in their work on the heart. 


\section{Materials and Methods}

\subsection{Segmentation of the Myocardium}

Let $\bar{C}_{n}$ represent a vector of Cine-DEMR images with $n$ consecutive phases describing one spatial slice of the heart. Let $S_{1}(r)$ represent a contour manually drawn on phase 1 .

\subsubsection{Localization of the $\mathrm{LV}$ in Phases 2..n}

The approximate position of the LV in phases $k=2 . . n$ is found through a non-rigid registration of $C_{1}$ with $C_{k}, k=2 . . n$. This results in a series of deformation fields $D_{k}$ such that

$$
C_{1}(\vec{x}) \mapsto C_{k,}\left(D_{k}(\vec{x})\right), k=2 . . n
$$

These deformation fields are then applied to $S_{1}(r), r: 0 \rightarrow 1$, to arrive at

$$
S_{\text {localized }_{k}}(r)=D_{k}\left(S_{1}(r)\right), k=2 . . n
$$

The centroid of $S_{\text {localized }_{i}}(r)$,

$$
\vec{x}_{\text {center }_{k}}=\int_{S_{\text {localized }_{k}}} S_{\text {localized }_{k}}(r) d r
$$

is the position from which we start our search for the $\mathrm{LV}$ in $C_{k}, k=2 . . n$. This deformation is too imprecise for any other inferences. The resulting contour is too deformed to serve as a starting point for the next step of the procedure. Rather, we simply copy over $C_{1}$ to $C_{k}$ and center it on $\vec{x}_{\text {center }_{k}}$ resulting in $S_{k}^{\prime}(r)$.

\subsubsection{Affine Transformation of Contours}

We deform $S_{k}^{\prime}(r)$ to fit the image $C_{k}$ by applying an affine deformation composed of 5 parameters: translation in the $\mathrm{x}$ and $\mathrm{y}$ dimensions $\vec{\tau}_{k}$, shearing parameters $s_{q_{k}}$ and $s_{m_{k}}$ in $H_{k}\left(s_{q_{k}}, s_{m_{k}}\right)=\left(\begin{array}{cc}1 & s_{q_{k}} \\ s_{m_{k}} & 1\end{array}\right)$, and a scaling parameter $\omega_{k}$. The translation is bound by the distance of 10 pixels, the shearing bound by \pm 60 degrees and scaling by $\pm \% 20$.

The affine parameters are adjusted to minimize an energy term made up of the components: $E_{1}, E_{2}$, and $E_{3}$ such that: 


$$
S_{k}^{\prime \prime}(r)=\underset{\tau_{k}, s_{q k}, s_{m_{k}}, \omega_{k}}{\operatorname{ArgMax}}\left[\begin{array}{l}
W_{1} \int E_{1}\left(\omega_{k} H\left(s_{q_{k}}, s_{m_{k}}\right) S_{k \text { endo }}^{\prime}(r)+\tau_{k}\right) d r+ \\
W_{2} \int E_{2}\left(\omega_{k} H\left(s_{q_{k}}, s_{m_{k}}\right) S_{k e p i}(r)+\tau_{k}\right) d r+W_{3} \oint E_{3}\left(\omega_{k} H\left(s_{q_{k}}, s_{m_{k}}\right) S_{k: p p i}^{\prime}(r)+\tau_{k}\right) d r
\end{array}\right]
$$

The resulting contour $S_{k}^{\prime \prime}(r)$ serves as a starting point for a local refinement process.

\subsubsection{Local Refinement of the Tracking}

Over the course of the cardiac cycle, the shape of the LV deforms in a non-rigid manner. The affine transformation from the previous section is capable of describing this only to a limited extent. However, it does provide a good basis from which to begin a less constrained search. Therefore, we apply an ASM-like approach to refining $S_{k}^{\prime \prime}(r)$.

Essentially, we determine the signatures of radial profiles in the neighborhoods of the inner and outer contours of $S_{1}(r)$. We then locally deform $S_{k}^{\prime \prime}(r)$ such that the signature of its profiles in the neighborhoods of the inner and outer contours match those of $S_{1}(r)$.

\subsubsection{Profile Calculation}

We sample both inner and outer contours in $j$ radial directions yielding profiles $\vec{p}_{k j_{\text {endo }}}(\rho)$ and $\vec{p}_{k j_{\text {epi }}}(\rho), k=1 . . n$. The radial directions are centered at the centroid, $\vec{\mu}_{k}$, of $S_{1 e p i}(r)$ or $S_{k_{e p i}}{ }^{\prime \prime}(r)$ (depending on k). Note: $\vec{\mu}_{k}$ may be different from $\vec{x}_{\text {center }_{k}}$ due to the affine transformation in Section 3.1.2). The rays intersect $S_{1}(r)$ or $S_{k}^{\prime \prime}(r)$ (depending on k) at $\vec{q}_{k j_{\text {endo }}}$ and $\vec{q}_{k j_{e p i}}$.

For a particular image $k$ (we drop the $k$ subscript here for clarity), profile $\vec{p}_{j}(\rho)$ (whether for the inner contour $\vec{p}_{j_{\text {endo }}}(\rho)$ or outer contour $\vec{p}_{j_{e p i}}(\rho)$ ) is a linear function of $\rho,-\frac{\kappa}{2} \leq \rho \leq \frac{\kappa}{2}$ centered at $\vec{q}_{j}$.

$$
\vec{p}_{j}(\rho)=\left(\left(\mu_{x}+\operatorname{rad}_{j}-\frac{\kappa}{2}+\rho\right) \operatorname{Cos} \phi_{j},\left(\mu_{y}+\operatorname{rad}_{j}-\frac{\kappa}{2}+\rho\right) \operatorname{Sin} \phi_{j}\right)
$$

where $\operatorname{rad}_{j}=\sqrt{\left(\vec{\mu}-\vec{q}_{j}\right)^{2}}, \vec{\mu}=\left(\mu_{x}, \mu_{y}\right)$, and $\kappa$ is the length of the profile.

The energy $\varepsilon_{\text {data }}$ for matching the profiles is expressed as a function of the offset $\vec{v}_{k j}$ from $\vec{q}_{k j}$ (returning to the $k$ subscript),

$$
\left.\varepsilon_{\text {data }}\left(\vec{v}_{k j}\right)=\int_{-\frac{\kappa}{2}}^{\frac{\kappa}{2}}\left(C_{k}\left(\vec{p}_{k j}(\rho)+\vec{v}_{k j}\right)-C_{1}\left(\vec{p}_{1 j}(\rho)\right)\right)^{2}\right) d \rho
$$


This energy is minimal where the profile in image $C_{k}$ is offset by $\vec{v}_{k j}$ best matches the profile in image $C_{1}$.

\subsubsection{Refining the Contours}

For the outer contour, the optimal offset is determined simply by minimizing $\mathcal{E}_{\text {data }}\left(\vec{v}_{k j_{e p i}}\right)$ with respect to $\vec{v}_{k j_{e p i}}$,

$$
\vec{v}_{k j_{e p i}}=\underset{\vec{v}_{k j e p i}^{\prime}}{\operatorname{ArgMin}} \mathcal{E}\left(\vec{v}_{k_{j e p i}}^{\prime}\right)
$$

For each $S_{k_{e p i}}$ " $(r)$, a cubic spline is fit to the points $\left(\vec{q}_{k j_{e p i}}+\vec{v}_{k j_{e p i}}\right)$, for all $j$, to form the final outer contour, $F_{k_{e p i}}(r)$.

The fitting of the inner contour is less stable and requires a smoothing coefficient. This coefficient represents a tethering of the inner contour to the fixed outer contour with a spring which limits its deformation. For a given image, $k=2 . . n$ (we drop the $k$ subscript here for clarity),

$$
\gamma\left(\vec{v}_{j_{\text {endo }}}\right)=1+\frac{\left|\operatorname{dist}\left(\vec{q}_{j_{\text {epi }}}^{\prime}-\vec{q}_{j_{\text {endo }}}\right)-\operatorname{dist}\left(\vec{q}_{j_{\text {epi }}}^{\prime}-\left(\vec{q}_{j_{\text {endo }}}+\vec{v}_{j_{\text {endo }}}\right)\right)\right|}{\operatorname{dist}\left(\vec{q}_{j_{\text {epi }}^{\prime}}^{\prime}-\vec{q}_{j_{\text {endo }}}\right)}
$$

Where $\vec{q}_{j_{e p i}}^{\prime}$ is the intersection in the radial direction with the final outer contour $F_{k_{e p i}}(r)$. Therefore, the optimal offset for the inner contour is determined by minimizing with respect to $\vec{v}_{k j_{\text {endo }}}$ (returning to the $k$ subscript),

$$
\vec{v}_{k j_{\text {endo }}}=\underset{\vec{v}_{k j_{\text {endo }}}^{\prime} \operatorname{Ain}}{\operatorname{Arg}}\left(\gamma\left(\vec{v}_{k j_{\text {endo }}}^{\prime}\right) \mathcal{E}\left(\vec{v}_{k j_{\text {endo }}}^{\prime}\right)\right)
$$

Similarly, a cubic spline is fit to the points $\left(\vec{q}_{k j_{e n d o}}+\vec{v}_{k j_{e n d o}}\right)$ to form the final contour $F_{k_{\text {endo }}}(r) \cdot$

\subsection{Classification of Tissues}

We employ an SVM to classify the myocardial pixels once the borders have been detected. For our kernel function of the SVM we use a Gaussian radial basis function of the form:

$$
k\left(\bar{\phi}(\vec{x}), \bar{\phi}\left(\vec{x}^{\prime}\right)\right)=e^{-\left\|\bar{\phi}(x)-\bar{\phi}\left(x^{\prime}\right)\right\|^{2} / 2 \sigma^{2}}
$$

where $\bar{\phi}$ is the vector of features. The feature vector $\bar{\phi}$ is made up of three components: First, the intensity of a pixel, relative to the average myocardial intensity. Second, the standard deviation of the relative pixel intensities with respect to its next neighbors. Finally, the "myocardial contrast" defined as the ratio of the mean myocardial intensity and the mean image intensity of the image. Training was 
performed on static DEMR images due to the lack of available Cine-DEMR examples. To determine $\sigma$ in our kernel as well as $K$, a compromise between maximizing the margin and minimizing the number of training set errors, we employed the "leave-one-out strategy".

\subsection{Acquisition Protocol}

Cine-DEMR imaging is based on inversion recovery, single-shot, balanced steady state free precession (bSSFP) imaging [Chung]. Each image frame of the ECGtriggered cine series is acquired during a separate RR-interval using a constant inversion time. Image frames are acquired every other heart beat to allow magnetization recovery. To create a cine series, the trigger delay is varied between image frames, resulting in a series of single-shot images, each from a different phase of the cardiac cycle.

Cine-DE images were acquired using a 1.5T MRI scanner (Magnetom Sonata, Siemens Medical Solutions, Erlangen, Germany), using the following typical acquisition parameters: flip angle $50^{\circ}$, repetition time $2.5 \mathrm{~ms}$, echo time $1.1 \mathrm{~ms}$, bandwidth $1090 \mathrm{~Hz} /$ pixel, field-of-view $380 \mathrm{~mm}$, rectangular field-of-view 75\%, acquisition matrix $192 \times 115$ (frequency, phase). By default, 15 image frames are acquired in each CINE-DE series, with a variable temporal spacing to cover the cardiac cycle. Images are acquired during a single breath-hold, approximately 10-20 minutes after intravenous injection of $40 \mathrm{ml}$ of $0.5 \mathrm{mmol} / \mathrm{ml}$ gadopentetate dimeglumine (Magnevist, Berlex Imaging, Wayne NJ).

\section{Results}

We validated our study by comparing our automated results with ground truth provided by an expert. Three phases (frames 5, 10, and 15) from each patient were collected for a total of 15 images. An expert different from the one providing ground truth supplied the initial segmentation to initialize the tracking (frame 1). Figure 2 (Left) shows an example frame.
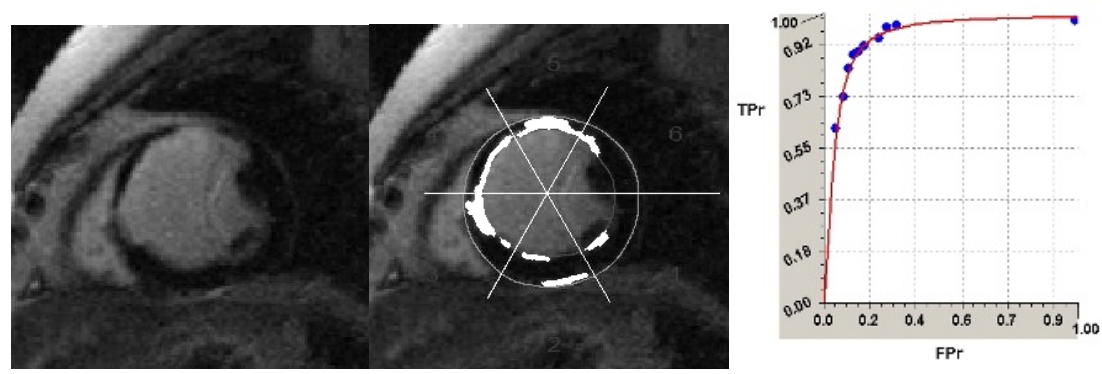

Fig. 2. Left: An example Cine-DEMR image. Middle: the tracking/classification protocol applied to the example image. The white pixels represent those found to be non-viable. Right: The ROC curve describing the results of our experiments. $96.80 \%$ of the area is under the curve. 
For the segmentation, 32 radial directions were used for the profiles. Both the inner and outer profiles had length $\kappa=5$ pixels and the search space for matching the profiles was 3 square pixels. The tracking of the myocardial borders had an average error of 2.1 pixels over all images. Regarding the classification of pixels as viable or non-viable, appropriate SVM parameters were found to be $\sigma=0.01$ and $K=20$. Our experiments had a sensitivity of $80.76 \%$, specificity $96.54 \%$ with $96.80 \%$ of the area covered under the ROC (see Figure 2 (Right)). The general correctness rate was $93.46 \%$.

\section{Discussion}

The tracking of the myocardial borders was performed from the first phase to every other phase $(1 \rightarrow n)$ rather than from one phase to the next $(n \rightarrow n+1)$. This approach was taken because the profile signatures in phase 1 were guaranteed to be correct (having been based on manually drawn contours) and were a better basis for matching. We initially attempted a consecutive frame $(n \rightarrow n+1)$ approach but found that a slight drift over the series resulted in poorer segmentations. Our scheme depends on the general appearance of the myocardium staying constant over the cardiac cycle. As mentioned in the introduction, this may not always be the case due to through plane motion. Therefore, we are currently investigating a Kalman filter approach to this tracking.

As described in Section 3.1.5, the refinement of the endocardial and endocardial contours is treated differently in the tracking. The endocardial border generally deforms much less significantly than the endocardial border over the cardiac cycle. Because the endocardial border is subject to such variability, we found that without the smoothing effect of tethering to the epicardium, the endocardial segmentation was far too jagged to appear natural. This tethering had a strong impact particularly when the tissue was very damaged. In these cases the myocardium was bright enough to be indistinguishable from the blood pool so there was little influence from the data energy $\mathcal{E}_{\text {data }}$. In these cases, the dead tissue moves very little since it is, in fact, dead. Therefore reliance on the tethering influence yielded good results.

Finally, as mentioned in the introduction, Cine-DEMR and DEMR have different resolutions and thus appear slightly dissimilar from one another. Our training set for the classification of the myocardial tissues was based on standard DEMR datasets since we had few Cine-DEMR datasets on which to train the SVM. We attribute our relatively low sensitivity in our results to the fact that the training set and testing sets do not come from the same population.

\section{Conclusions}

We have presented preliminary findings on the tracking and classification of a new MR imaging sequence Cine-DEMR. In the future we hope to develop methods for the segmentation of the first phase of these series thereby creating a fully automatic quantification procedure. 


\section{References}

[Cootes] T. Cootes and C. Taylor, "Statistical Models of Appearance for Computer Vision" October 26, 2001, pp 37-43, available at www.isbe.man.ac.uk.

[Cristianini] Cristianini N, Shawe-Taylor J. An Introduction to Support Vector Machines and other kernel-based learning methods. Cambridge University Press 2000.

[El-Naqa] El-Naqa I,, Yongyi Yang, Miles N. Wernick, Nikolas P. Galatsanos, and Robert Nishikawa, "Support Vector Machine Learning for Detection of Microcalcififcations in Mammograms", IEEE ICIP, Rochester, NY, September 2002

[Fieno] Fieno DS, Kim RJ,et.al.,. Contrast enhanced MRI of myocardium at risk: distinction between reversible and irreversible injury throughout infarct healing. 2000;36:1985-1991.

[Kim] R.J. Kim, D.S. Fieno, et. al., "Relationship of MRI Delayed Contrast Enhancement to Irreversible Injury, Infarct Age, and Contractile Function”, Circulation 1999; 100: 1992-2002.

[Dikici] Engin Dikici, T. O’Donnell. et. al.,.., “Quantification of Delayed Enhancement Images", Proceedings of MICCAI 2004.

[Schwartzman] Schwartzman PR., White RD. Magnetic Resonance Imaging. In: Topol EJ, ed. Textbook of Cardiovascular Medicine, 2nd edition. Philadelphia: LippincottRaven, 2002.

[Kim NEJM] Kim RJ, Wu E, Rafael A, Chen EL, Parker MA, Simonetti O, Klocke FJ, Bonow RO, Judd RM. The Use of Contrast-Enhanced Magnetic Resonance Imaging to Identify Reversible Myocardial Dysfunction. N Engl J Med 2000; 343:1445-53.

[Setser] Setser RM, Chung YC, Kim JK, Stillman AE, Simonetti OP, Weaver JA, White RD. Cine Delayed Enhancement Imaging of the Heart. J Cardiovascular Magn Reson 2002; 7(1): 298-299 (Abstract).

[Chung] Chung YC, Vargas J, Simonetti O. Infarct imaging in a single heart beat. J Cardiovasc. Magn Reson 2002; 4: 12 (Abstract)

[Mitchell] S.C. Mitchell, J.G. Bosch, et. al., "3D Active Appearance Models: Segmentation of Cardiac MR and Ultrasound images." IEEE TMI vol. 20(9), 2002

[Noble] Noble N., Hill, D.. et. al., "The Automatic Identification of Hibernating Myocardium", MICCAI 2004, pgs. 890-898.

[Sun] Sun, Y, Jolly, MP. Moura J, "Contrast Invariant Registration of Cardiac and Renal MR Perfusion Images”, MICCAI04 905-910. 